\title{
A simple immunohistochemical panel comprising 2 conventional markers, Ki67 and p53, is a powerful tool for predicting patient outcome in luminal-type breast cancer
}

Takayuki Kobayashi ${ }^{1 *}$, Keiichi Iwaya', Tomoyuki Moriya², Tamio Yamasaki², Hitoshi Tsuda ${ }^{3}$, Junji Yamamoto ${ }^{2}$ and Osamu Matsubara'

\begin{abstract}
Background: Ki67 is widely used in order to distinguish the " $A$ " and " $B$ " subtypes of luminal-type breast cancer. This study aimed to validate the prognostic value of adding p53 to Ki67 for characterizing luminal-type breast cancer.

Methods: Immunostaining for Ki67, p53, and the molecular markers HER2, CK5/6, CK14, EGFR, FOXA1, GATA3, and P-cadherin was examined hormone receptor (HR)-positive cancer tissues from 150 patients. The prognostic value of an immunohistochemical panel comprising Ki67 and p53 was compared with that of the single Ki67 labeling index (LI), and uni- and multivariate analyses were performed.

Results: Division of the patients based on the immunohistochemistry results into favorable- (low Ki67 LI, p53negative) and unfavorable- (high Ki67 LI and/or p53-positive) phenotype groups yielded distinctly different KaplanMeier's curves of both disease-free $(P<0.0001)$ and overall survival $(P=0.0007)$. These differences were much more distinct than those between the corresponding low Ki67 LI vs. high Ki67LI curves. While the prognostic values of the other molecular markers were not significant, combined Ki67-p53 status was an independent prognostic factor by multivariate analysis.
\end{abstract}

Conclusion: These data indicate that an immunohistochemical panel comprising Ki67 and p53 is a practical tool for management of patients with HR-positive breast cancer.

Keywords: Ki67 labeling index, p53, IHC panel, Luminal-type breast cancer

\section{Background}

Approximately $70 \%$ of breast cancers express a hormone receptor (HR). HR status is a powerful predictor of response to therapies that inhibit estrogen synthesis or block the action of its receptor [1]. Endocrine therapies are established in the adjuvant setting [2-4]. For example, women with node-negative, HR-positive breast cancer who are treated with tamoxifen alone after surgery have an average 10-year recurrence rate of only $15 \%$ [5]. If all of these patients were offered chemotherapy, $85 \%$ would be over-treated [6]. It is therefore

\footnotetext{
*Correspondence: tkobayashi.g@gmail.com

'Department of Basic Pathology, National Defense Medical College, 3-2 Namiki, Tokorozawa, Saitama 359-8513, Japan

Full list of author information is available at the end of the article
}

important to distinguish patients with HR-positive tumors at high risk for recurrence who need additional chemotherapy from those for whom adjuvant hormonal therapy alone may suffice [7].

Multi-gene assays are strong candidate tools for predicting the risk of recurrence in HR-positive patients. For example, the Oncotype $\mathrm{DX}^{\mathrm{TM}}$ assay analyzes the expression levels of 21 genes (including 5 reference genes) in formalin-fixed paraffin embedded tissues and produces a Recurrence Score (RS) that predicts the likelihood of distant recurrence [6] and the benefit of chemotherapy in women with early HR-positive breast cancer [8]. Although Oncotype $\mathrm{DX}^{\mathrm{TM}}$ is a potentially powerful tool for stratification of HR-positive patients, it is too expensive to use in routine clinical practice. Many 
oncologists are eager for an alternative assay that is inexpensive as well as easy to use; one possible approach would be an immunohistochemical (IHC) assay.

Sorlie et al. reported that breast cancers could be divided, based on their gene expression profiles, into at least 4 groups: luminal-type, HER2-type, normal-liketype, and basal-type. Luminal-type cancers are characterized by an activated estrogen receptor (ER) signaling pathway and are divided into 2 subtypes, luminal subtypes " $A$ " and "B. In general, luminal-subtype-A tumors express higher levels of ER and carry a better prognosis than do luminal-subtype-B tumors [9]. Recent studies have shown that tumors of luminal subtype A have a lower rate of $p 53$ mutation [9-12] and are less proliferative $[13,14]$ than those of luminal subtype $B$, suggesting that the combination of p53 status and proliferation markers could be useful to distinguish between luminal subtypes $A$ and $B$.

The tumor suppressor gene $p 53$ plays a most important role in regulating normal cell fate in response to various stresses, and disruption of p53 function is often involved in tumor progression. Since the co-authors first reported in 1991 that distinct immunoreaction with p53 in the nuclei of breast cancer cells is an independent prognostic indicator [15], more than 1000 articles about the correlation between p53 status and breast cancer prognosis have been published. Recent studies have shown that abnormalities of the $p 53$ gene [16] and accumulation of p53 protein in the nuclei $[17,18]$ are also robust prognostic indicators in HRpositive patients.

Ki67 is the marker most often used to evaluate tumor proliferation status. The Ki67 protein is a large (395 kD) nuclear protein that is present during all active phases of the cell cycle except for the G0 phase. Because proliferation status is closely correlated with tumor aggressiveness, the Ki67 labeling index (LI) is considered an established prognostic marker for various tumor types, including breast cancer $[19,20]$. Previous clinical studies have revealed Ki67 LI to be a good prognostic indicator for HR-positive breast cancer patients [7,21].

Although Ki67 is a strong prognostic indicator for HR-positive breast cancer patients, adding Ki67 to the commonly used indices in daily practice is controversial [19]. Furthermore, its predictive value is weaker than that of multi-gene expression assays such as Oncotype $\mathrm{DX}^{\mathrm{TM}}$. In this study, we attempted to validate the classification of HR-positive breast cancer patients by combined analysis of Ki67 LI and p53 status. We performed immunohistochemical examination of Ki67 and p53 expression in 150 samples of surgically resected HR-positive invasive breast cancers and analyzed the relationships between combined Ki67-p53 status and clinicopathological factors, including prognosis.

\section{Methods}

\section{Patients and Samples}

Of the 247 patients who had undergone mastectomy or breast-conserving surgery for invasive ductal carcinoma of the breast at the National Defense Medical College (NDMC) Hospital between 1995 and 1999, 150 patients with ER-positive and/or progesterone-receptor (PgR)positive localized breast carcinomas were selected based on immunohistochemical reevaluation of ER and PgR expression. Tissue microarray (TMA) blocks of the tumors from these 150 patients were constructed as previously described [22]. Briefly, double tissue cores $2 \mathrm{~mm}$ in diameter were taken from each donor block, and these core specimens were transferred to a recipient block using a Tissue Microarrayer (Beecher Instruments, Silver Spring, MD, USA). The use of the tissue blocks was internally reviewed and approved by the NDMC Ethics Committee.

The 150 patients had been followed up for a median of 82 months (range, 1-151 months), during which time there were 30 relapses and 15 deaths. In most cases, the patients were prescribed adjuvant endocrine therapy (for example, tamoxifen, toremifene, fadrozole, or LHRH analogues). Forty-nine patients with large tumors and/or 4 or more lymph node metastases had received adjuvant chemotherapy (cyclophosphamide-epirubicin-5-fluorouracil (CEF), cyclophosphamide-adriamycin-5-fluorouracil (CAF), cyclophosphamide-methotrexate-5-fluorouracil (CMF), or oral fluoropyrimidines), and 12 patients with locally advanced breast cancer had received preoperative chemotherapy (for example, CAF or CEF). One hundred forty-eight patients were females and 2 were males. The clinical stage of the patients was determined based on the TNM classification according to general rules of the Japanese Breast Cancer Society [23]. Clinicopathological data were obtained from the medical records and pathology reports, but ER, PgR and HER2 status were examined in our previous study [22].

\section{Immunohistochemistry}

Immunohistochemistry was performed on a TMA composed of 150 breast cancer tissue specimens. The antibodies used were mouse monoclonal anti-p53 antibody (DO-7, Dako, Glostrup, Denmark), mouse monoclonal anti-Ki67 antibody (MIB-1, Dako), mouse monoclonal anti-FOXA1 antibody (2D7, Abnova, Taipei, Taiwan), mouse monoclonal anti-GATA3 antibody (HG3-31, Santa Cruz, Santa Cruz, CA, USA) mouse monoclonal anti-CK5/6 antibody (D5/16 B4, Dako), mouse monoclonal anti-CK14 antibody (LL002, NeoMarkers, Fremont, CA, USA), mouse monoclonal antiP-cadherin antibody (56C1, Novocastra, Newcastle, UK), and a mouse monoclonal anti-EGFR antibody included in an EGFR pharmDX kit (Dako). 
Sections (4-um-thick) were cut from the formalinfixed, paraffin-embedded TMA blocks. Antigens were retrieved by microwave heating for $30 \mathrm{~min}$ in $10 \mathrm{mM}$ sodium citrate ( $\mathrm{pH}$ 6.0) for CK5/6 and GATA3 or by autoclaving for $20 \mathrm{~min}$ in $10 \mathrm{mM}$ Tris- $\mathrm{HCl}(\mathrm{pH}$ 9.0) for Ki67, p53, CK14, FOXA1, and P-cadherin. To block endogenous peroxidase activity, the sections were treated for 5 min with $100 \%$ methanol containing $3 \% \mathrm{H}_{2} \mathrm{O}_{2}$. Non-specific binding was blocked by incubation in $1 \%$ normal swine serum (Dako) in phosphate-buffered saline. The slides were incubated with primary antibodies at $4^{\circ} \mathrm{C}$ overnight and then reacted with a dextran polymer reagent combined with secondary antibodies and peroxidase (Envision Plus; Dako) for $30 \mathrm{~min}$ at room temperature. Specific antigen-antibody reactions were visualized with $0.2 \%$ diaminobenzidine tetrahydrochloride and hydrogen peroxide. Immunostaining for EGFR was performed in accordance with the package inserts of the EGFR pharmDX Kit. The sections were counterstained with Mayer's hematoxylin.

\section{Evaluation of immunohistochemistry}

Although there is no universal cut-off value for Ki67 LI, Cheang et al. showed that, using the cases which were subtyped by gene expression profile, the best Ki67 LI cut-off value to distinguish luminal B from luminal A was $13 \%$ [7]. Furthermore, similar to the $10 \%$ cut-off value was used in several reports [21,24-28]. So, in this study, Ki67 LI greater than 10\% was classified as high. The Ki67 LI was calculated as the percentage of positive tumor nuclei divided by the total number of tumor cells examined on the basis of a manual count of 500 or more cells under high power $(400 \times)$.

For p53, FOXA1, and GATA3, cells with immunostaining in the nucleus were defined as positive, while for CK5/6, CK14, and P-cadherin, cells with immunostaining along the cellular periphery and/or in the cytoplasm were defined as positive. For p53, positive staining of fewer than $10 \%$ of the tumor cells was defined as negative tumor expression and staining of $10 \%$ or more of the tumor cells as positive tumor expression [15]. For Pcadherin, membrane staining of fewer than $50 \%$ of the tumor cells was defined as negative tumor expression and staining of $50 \%$ or more of the tumor cells as positive tumor expression. P-cadherin positive tumors were further divided into "weakly" and "strongly" expressing tumors based on staining intensity. Finally, negative and weakly P-cadherin-staining tumors were classified as

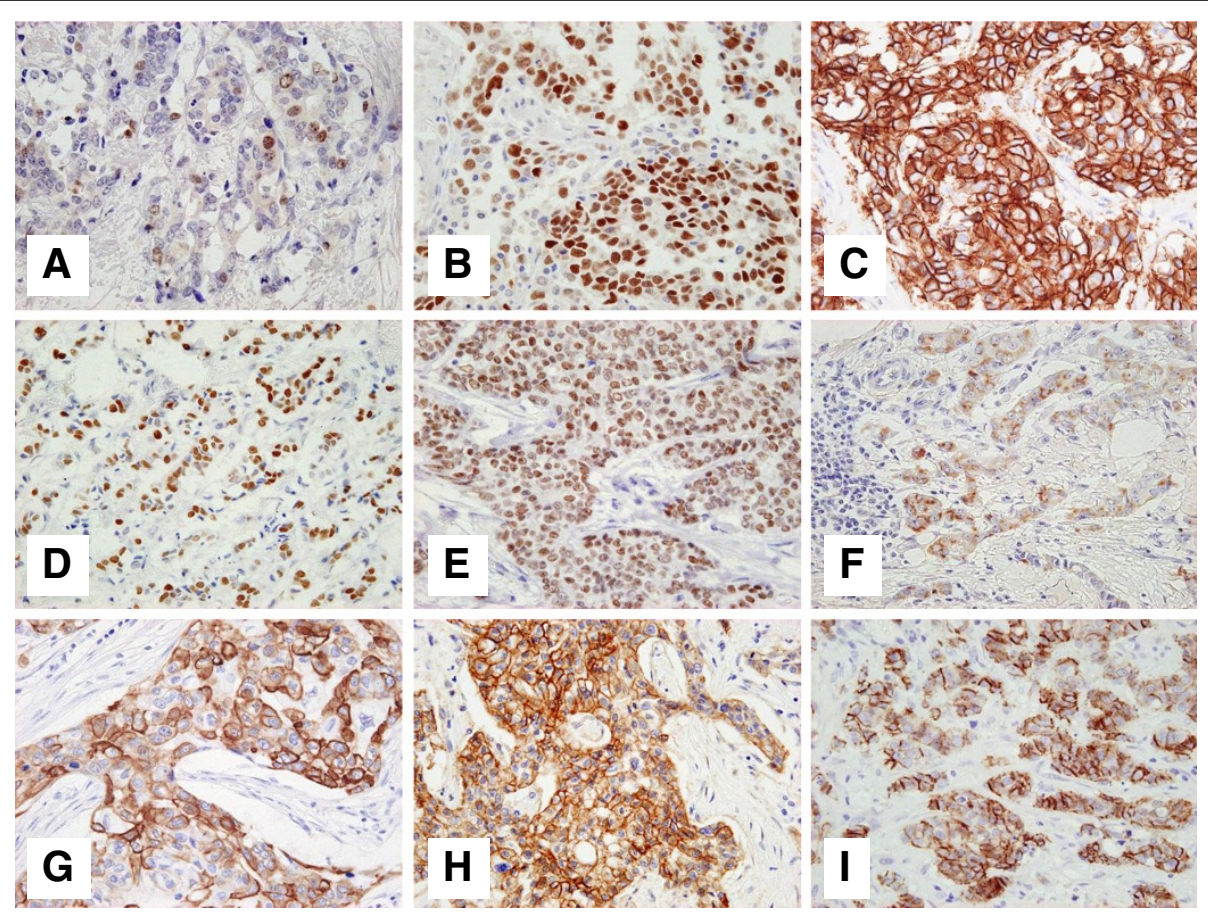

Figure 1 Representative images of immunostaining for 9 molecular markers. A, Positive nuclear Ki67 staining. The Ki67 labeling index of this specimen is $12 \%$. B, p53 staining in the nucleus. This tumor was scored as p53-positive. C, Positive HER2 membrane staining. The HER2 expression of this tumor was scored as 3+. D, Positive nuclear FOXA1 staining. This tumor was classified as FOXA1-positive. E, Positive nuclear GATA3 staining. This tumor was classified as GATA3-positive. $\mathbf{F}$, Positive CK5/6 membrane or sub-membrane staining of tumor cells. This tumor was classified as CK5/6-positive. G, Positive CK14 membrane or sub-membrane staining of tumor cells. This tumor was classified as CK14-positive. $\mathbf{H}$, Positive EGFR membrane staining of tumor cells. The EGFR expression of this tumor was scored as 3+. I, Positive P-cadherin membrane or submembrane immunoreactivity of tumor cells. This tumor was classified as P-cadherin-high. The magnification of all figures is $\times 400$. 
Table 1 Clinicopathological implication of Ki67-p53 combination status in surgically resected hormone receptorpositive breast cancers

Cases

\begin{tabular}{|c|c|c|c|c|}
\hline \multirow{4}{*}{ Variables } & \multicolumn{4}{|c|}{ Cases } \\
\hline & \multicolumn{4}{|c|}{ Ki67-p53 combination status } \\
\hline & Total & Low Ki67 Lland Negative p53 & High Ki67 LI and/or Positive p53 & $P$ - value \\
\hline & $(n=150)$ & $(n=88)$ & $(n=62)$ & \\
\hline \multicolumn{5}{|l|}{ Age } \\
\hline$\leqq 50$ & 71 & 40 & 31 & \\
\hline$>50$ & 79 & 48 & 31 & 0.58 \\
\hline \multicolumn{5}{|l|}{ Tumor size } \\
\hline$<5.0 \mathrm{~cm}$ & 128 & 78 & 50 & \\
\hline$\geqq 5.0 \mathrm{~cm}$ & 19 & 8 & 11 & 0.12 \\
\hline Unknown & 3 & 2 & 1 & \\
\hline \multicolumn{5}{|c|}{ Lymph node metastasis } \\
\hline$(-)$ & 84 & 52 & 32 & \\
\hline$(+)$ & 63 & 34 & 29 & 0.33 \\
\hline Unknown & 3 & 2 & 1 & \\
\hline \multicolumn{5}{|l|}{ Stage } \\
\hline | or || & 129 & 78 & 51 & \\
\hline III & 17 & 7 & 10 & 0.13 \\
\hline Unknown & 4 & 3 & 1 & \\
\hline \multicolumn{5}{|l|}{ Nuclear grade } \\
\hline 1,2 & 115 & 76 & 39 & \\
\hline 3 & 35 & 12 & 23 & 0.0008 \\
\hline \multicolumn{5}{|l|}{ HER2 status } \\
\hline Negative & 142 & 88 & 54 & \\
\hline Positive & 8 & 0 & 8 & 0.0006 \\
\hline \multicolumn{5}{|c|}{ Basal phenotype marker (CK5/6, CK14, EGFR) } \\
\hline Negative & 140 & 87 & 53 & \\
\hline Positive & 10 & 1 & 9 & 0.0016 \\
\hline \multicolumn{5}{|l|}{ FOXA1 } \\
\hline Negative & 20 & 14 & 6 & \\
\hline Positive & 127 & 72 & 55 & 0.38 \\
\hline NE & 3 & 2 & 1 & \\
\hline \multicolumn{5}{|l|}{ GATA3 } \\
\hline Negative & 30 & 18 & 12 & \\
\hline Positive & 120 & 70 & 50 & 0.99 \\
\hline \multicolumn{5}{|l|}{ P-cadherin } \\
\hline Low & 94 & 62 & 32 & \\
\hline High & 56 & 26 & 30 & 0.0019 \\
\hline \multicolumn{5}{|l|}{ Chemotherapy } \\
\hline No & 98 & 59 & 39 & 0.59 \\
\hline Yes & 52 & 29 & 23 & \\
\hline
\end{tabular}

Abbreviation: $L /$ labeling index, NE not evaluable. 

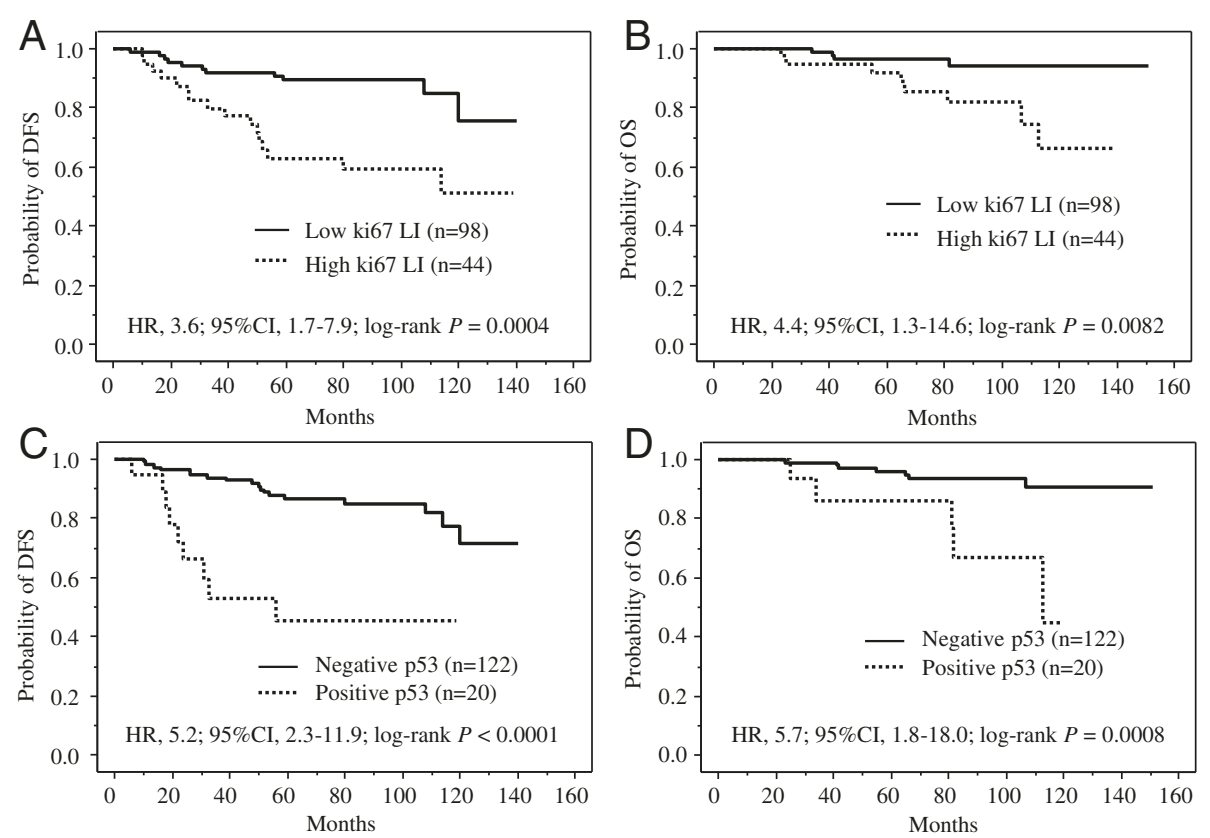

Figure 2 Prognostic impact of Ki67 labeling index (LI) and p53 status in patients with hormone receptor-positive and HER2-negative breast cancer $(\mathbf{n}=\mathbf{1 4 2})$. A, Disease free survival curves for 98 patients with Ki67 LI-low tumors and 44 patients with Ki67 LI-high tumors. The 2 curves differ significantly $(\mathrm{HR}, 3.6 ; 95 \% \mathrm{Cl}, 1.7-7.9$; $\log$-rank $P=0.0004)$. B, Overall survival curves for 98 patients with Ki67 LI-low tumors and 44 patients with Ki67 LI-high tumors. The 2 curves differ significantly (HR, 4.4; 95\% Cl, 1.3-14.6; log-rank $P=0.0082)$. C, Disease-free survival curves for 122 patients with p53-negative tumors and 20 patients with p53-positive tumors. The 2 curves differ significantly (HR, 5.2; 95\% Cl, 2.3-11.9; logrank $P<0.0001)$. D, Overall survival curves for 122 patients with p53-negative tumors and 20 patients with p53-positive tumors. The 2 curves differ significantly $(\mathrm{HR}, 5.7 ; 95 \% \mathrm{Cl}, 1.8-18.0$; $\log$-rank $P=0.0008)$.

"low" and strongly P-cadherin-staining tumors as "high". A tumor expression cutoff point of $10 \%$ of cells stained was used for GATA3, CK5/6, and CK14 and a cutoff of $70 \%$ of cells stained for FOXA1, regardless of staining intensity. An EGFR score of 0-3+ was assigned according to the manufacturer's package insert, and scores of $1-3+$ were classified as positive. CK5/6, CK14, and EGFR were considered basal phenotype markers.

ER and PgR were examined immunohistochemically as described in the previous study [22], using mouse monoclonal anti-human ER (clone 1D5, Dako) and mouse anti-human PgR (clone PgR636, DAKO). ER and PgR were defined as positive if the nuclear staining was seen in $10 \%$ or more of tumor cells. Hormone receptor positive was defined as at least one of ER or PgR positive, and hormone receptor negative was defined as ER and PgR negative. HER2 was evaluated by IHC using rabbit polyclonal anti-HER2 antibody (HercepTest kit, Dako) and FISH (in case of IHC 2+) using Path Vysion kit (Abbott Park, IL, USA). HER2 was defined as positive if the IHC score was " $3+$ " according to the standard procedure, or gene amplification (HER2:CEP17 ratio > 2.0) was detected by FISH [29].

The immunohistochemistry results were evaluated independently by 2 observers (TK and KI), and cases with disparate scores were re-evaluated and discussed until a consensus was reached. Ki67-positive cells were counted and the labeling index calculated by TK alone.

\section{Statistical analysis}

Comparisons between groups were evaluated with the chi-squared test or Fisher's exact test. Patient survival curves were drawn using the Kaplan-Meier method and analyzed by the log-rank test. The hazard ratios and corresponding 95\% confidence intervals (CIs) were calculated with Cox's proportional hazards model. Univariate and multivariate Cox's proportional hazards models were used to explore the associations of variables with disease-free and overall survival. For all tests, differences at $P<0.05$ were considered statistically significant. All analyses were performed using the software JMP 6.0 for Windows (SAS Institute Inc., Cary, NC, USA).

\section{Results}

Expression of markers (Ki67, p53, HER2, FOXA1, GATA3, CK5/6, CK14, EGFR, and P-cadherin) in HR-positive tumors $(n=150)$

Representative images of immunostaining for the markers examined in this study are shown in Figure 1. Among 150 HR-positive tumors, there were 51 (34\%) Ki67 LI-high tumors, 22 (15\%) p53-positive tumors, 127 (85\%) FOXA1-positive tumors, 120 (80\%) GATA3- 
Table 2 Univariate and multivariate analyses of immunohistochemical parameters (disease-free survival and overall survival)

\begin{tabular}{|c|c|c|c|c|c|c|c|c|}
\hline & & \multirow[b]{2}{*}{ Total $(n=142)$} & \multicolumn{3}{|c|}{ Univariate } & \multicolumn{3}{|c|}{ Multivariate } \\
\hline & & & Hazard ratio & $(95 \% \mathrm{Cl})$ & $P$-value & Hazard ratio & $(95 \% \mathrm{Cl})$ & $P$-value \\
\hline \multicolumn{9}{|l|}{ Disease-free survival } \\
\hline \multirow[t]{2}{*}{ Ki67 LI } & Low & 98 & 1 & & & 1 & & \\
\hline & High & 44 & 3.6 & $1.7-7.9$ & 0.0010 & 3.2 & $1.4-7.6$ & 0.0073 \\
\hline \multirow[t]{2}{*}{ p53 } & Negative & 122 & 1 & & & 1 & & \\
\hline & Positive & 20 & 5.2 & 2.3-11.9 & $<0.0001$ & 3.9 & $1.6-9.4$ & 0.0025 \\
\hline \multirow[t]{2}{*}{ FOXA1 } & Low & 20 & 1 & & & 1 & & \\
\hline & High & 119 & 1.4 & $0.51-3.6$ & 0.54 & 1.7 & $0.56-5.2$ & 0.34 \\
\hline \multirow[t]{2}{*}{ GATA3 } & Negative & 30 & 1 & & & 1 & & \\
\hline & Positive & 112 & 1.5 & $0.65-3.5$ & 0.34 & 1.2 & $0.46-3.4$ & 0.66 \\
\hline Basal phenotype marker & Negative & 133 & 1 & & & 1 & & \\
\hline (CK5/6, CK14, EGFR) & Positive & 9 & 1.0 & $0.24-4.3$ & 0.98 & 0.47 & $0.10-2.2$ & 0.34 \\
\hline \multirow[t]{2}{*}{ P-cadherin } & Low & 90 & 1 & & & 1 & & \\
\hline & High & 52 & 1.3 & $0.59-2.8$ & 0.52 & 0.87 & $0.37-2.1$ & 0.75 \\
\hline \multicolumn{9}{|l|}{ Overall survival } \\
\hline \multirow[t]{2}{*}{ Ki67 LI } & Low & 98 & 1 & & & 1 & & \\
\hline & High & 44 & 4.4 & $1.3-14.6$ & 0.016 & 3.2 & $0.91-11.9$ & 0.070 \\
\hline \multirow[t]{2}{*}{ p53 } & Negative & 122 & 1 & & & 1 & & \\
\hline & Positive & 20 & 5.7 & 1.8-18.0 & 0.0029 & 3.8 & $1.1-13.0$ & 0.030 \\
\hline \multirow[t]{2}{*}{ FOXA1 } & Low & 20 & 1 & & & 1 & & \\
\hline & High & 119 & 0.49 & $0.06-3.9$ & 0.50 & 0.75 & $0.07-7.5$ & 0.81 \\
\hline \multirow[t]{2}{*}{ GATA3 } & Negative & 30 & 1 & & & 1 & & \\
\hline & Positive & 112 & 1.1 & $0.31-4.3$ & 0.84 & 1.3 & $0.29-6.1$ & 0.70 \\
\hline Basal phenotype marker & Negative & 133 & 1 & & & 1 & & \\
\hline (CK5/6, CK14, EGFR) & Positive & 9 & 1.2 & $0.15-9.3$ & 0.86 & 0.60 & $0.07-5.4$ & 0.64 \\
\hline \multirow[t]{2}{*}{ P-cadherin } & Low & 90 & 1 & & & 1 & & \\
\hline & High & 52 & 1.8 & $0.57-5.5$ & 0.32 & 1.2 & $0.36-4.2$ & 0.73 \\
\hline
\end{tabular}

Abbreviation: 95\% Cl 95\% confidence interval, Ll labeling index.

positive tumors, and 6 (4\%) CK5/6-positive, 3 (2\%) CK14-positive, 3 (2\%) EGFR-positive, and 56 (37\%) Pcadherin-high tumors. Ten (7\%) tumors showed positive staining for at least 1 of the basal phenotype markers CK5/6, CK14, and EGFR. Eight tumors were determined as HER2-positive, which were composed of 6 tumors with IHC $3+$ and 2 tumors with IHC $2+$ and FISH +.

Correlations of clinicopathological factors (tumor size, lymph-node status, nuclear grade, and molecular markers) with Ki67 LI status and p53 immunoreactivity in HR-positive tumors $(n=150)$

The tumors with high Ki67 LIs showed significantly higher frequencies of high nuclear grade, HER2 positivity, basal phenotype marker positivity, and $\mathrm{P}$-cadherin $(P=0.013, P=0.0010$, and $P=0.0015$, and $P=0.013$, respectively). The tumors with positive p53 staining showed significantly higher frequencies of large tumor size and high nuclear grade $(P=0.0013$ and $P=0.035$, respectively).

\section{Correlation between clinicopathological factors and} combined Ki67-p53 status in HR-positive tumors $(n=150)$ There were 88 (59\%), 11 (7\%), 40 (27\%), and $11(7 \%)$ tumors with the Ki67 LI-low and p53-negative, Ki67 LIlow and p53-positive, Ki67 LI-high and p53-negative, and Ki67 LI-high and p53-positive phenotypes, respectively. The tumors with the "favorable" Ki67 LI-low and p53-negative phenotype $(n=88)$ showed lower frequencies of high nuclear grade, HER2 positivity, basal phenotype marker positivity, and high $\mathrm{P}$-cadherin expression $(P=0.0008, P=0.0006, P=0.0016$ and $P=0.0019$, respectively; Table 1) than did those with "unfavorable" Ki67 LI-low and p53-positive ( $\mathrm{n}=11)$, Ki67 LI-high and 

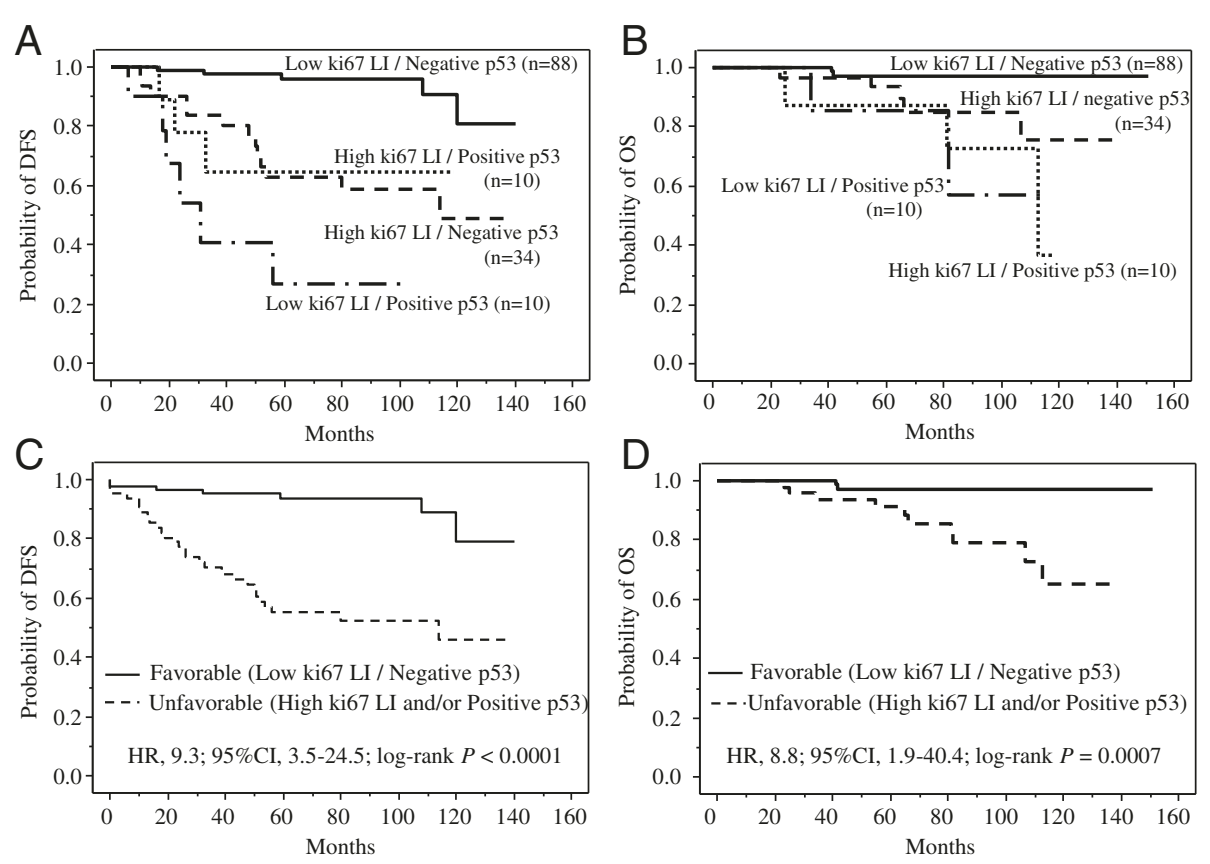

Figure 3 Prognostic impact of combined Ki67-p53 status in patients with hormone receptor-positive and HER2-negative breast cancer ( $\mathbf{n = 1 4 2 ) . ~ A , ~ D i s e a s e - f r e e ~ s u r v i v a l ~ c u r v e s ~ f o r ~} 88$ patients with Ki67 LI-low and p53-negative tumors, 34 patients with Ki67 LI-high and p53-negative tumors, 10 patients with Ki67 LI-low and p53-positive tumors, and 10 patients with Ki67 LI-high and p53-positive tumors. Patients with Ki67 LI-low and p53-negative tumors had significantly longer disease-free survival than those with Ki67 LI-low and p53-positive tumors, those with Ki67 LIhigh and p53-negative tumors, or those with Ki67 LI-high and p53-positive tumors $(P<0.0001, P<0.0001$, and $P=0.0005$, respectively). B, Overall survival curves for 88 patients with Ki67 LI-low and p53-negative tumors, 34 patients with Ki67 LI-high and p53-negative tumors, 10 patients with Ki67 LI-low and p53-positive tumors, and 10 patients with Ki67 LI-high and p53-positive tumors. Patients with Ki67 LI-low and p53negative tumors had significantly longer disease-free survival than those with Ki67 LI-low and p53-positive tumors, those with Ki67 LI-high and p53-negative tumors, or those with Ki67 LI-high and p53-positive tumors $(P=0.0010, P=0.011$, and $P<0.0001$, respectively). C, Disease-free survival curves for patients with favorable-phenotype tumors (88 patients with Ki67 LI-low and p53-negative tumors) and unfavorable-phenotype tumors (54 patients with Ki67 LI-high and/or p53-positive tumors). The disease-free survival time was significantly longer in the favorablephenotype group than in the unfavorable-phenotype group (HR, 9.3; 95\% Cl, 3.5-24.5; $P<0.0001)$. D, Overall survival curves for patients with favorable-phenotype tumors (88 patients with Ki67 LI-low and p53-negative tumors) and those with unfavorable-phenotype tumors (54 patients with Ki67 LI-high and/or p53-positive tumors). The disease-free survival was significantly longer in the favorable-phenotype group than in the unfavorable-phenotype group (HR, 8.8; 95\% Cl, 1.9-40.4; $P=0.0007)$.

p53-negative $(\mathrm{n}=40)$, and Ki67 LI-high and p53positive $(\mathrm{n}=11)$ phenotypes $(\mathrm{n}=62$ total). Interestingly, all HER2-positive tumors were shown to be unfavorable phenotype tumors. This study found no correlations between the combined Ki67-p53 status and the clinical factors tumor size, nodal status.

\section{Prognostic implications of combined Ki67-p53 status in HR-positive and HER2-negative tumors $(n=142)$}

The patients with HER2-positive tumors could be received anti-HER2 treatments which have tremendous effect in both adjuvant and metastatic setting [30,31]. So, we next conducted the further analyses using the cases with HR-positive and HER2-negative tumors in order to evaluate more definitely the clinical implication of Ki67 and p53.

Both the disease-free survival (DFS) and overall survival (OS) curves differed significantly between the patients with Ki67 LI-low tumors and those with Ki67-
LI-high tumors (DFS: HR, 3.6; 95\% CI, 1.7-7.9; log-rank $P=0.0004$; Figure 2A, and OS: HR, 4.4; 95\% CI, $1.3-$ 14.6; $\log$-rank $P=0.0082$; Figure $2 \mathrm{~B}$ ). Both curves also differed significantly between the patients with p53negative tumors and those with p53-positive tumors (DFS: HR, 5.2; 95\% CI, 2.3-11.9; log-rank $P<0.0001$; Figure $2 \mathrm{C}$, and OS: HR, 5.7; 95\% CI, 1.8-18.0; log-rank $P=0.0008$; Figure 2D). Furthermore, a multivariate analysis using Cox's proportional hazard model and including immunostaining for the markers Ki67, p53, HER2, FOXA1, GATA3, the basal phenotype markers, and P-cadherin selected Ki67 and p53 as significant prognostic factors for DFS $(P=0.0073$ and $P=0.0025$, respectively; Table 2$)$ and $\mathrm{p} 53$ for OS $(P=0.030$, Table 2$)$.

Figures $3 \mathrm{~A}$ and $3 \mathrm{~B}$ show the DFS and OS curves for the 4 combined Ki67-p53 status groups. Patients with Ki67 LI-low and p53-negative tumors survived longer than those in the other 3 groups (the patients with Ki67 LI-low and p53-positive tumors, Ki67-high and p53- 
Table 3 Univariate and multivariate analyses of disease-free survival in patients with hormone receptor-positive/HER2negative primary breast cancer

\begin{tabular}{|c|c|c|c|c|c|c|c|c|}
\hline & & \multirow[b]{2}{*}{ Total $(n=142)$} & \multicolumn{3}{|c|}{ Univariate } & \multicolumn{3}{|c|}{ Multivariate } \\
\hline & & & Hazard ratio & $(95 \% \mathrm{Cl})$ & $P$-value & Hazard ratio & $(95 \% \mathrm{Cl})$ & $P$-value \\
\hline \multirow[t]{2}{*}{ Ki67-p53 } & Low Ki67 LI and Negative p53 & 88 & 1 & & & 1 & & \\
\hline & High Ki67 LI and/or Positive p53 & 54 & 9.3 & $3.5-24.5$ & $<0.0001$ & 11.6 & $4.2-32.3$ & $<0.0001$ \\
\hline \multirow[t]{2}{*}{ FOXA1 } & Low & 20 & 1 & & & & & \\
\hline & High & 119 & 1.4 & $0.51-3.6$ & 0.54 & & & \\
\hline \multirow[t]{2}{*}{ GATA3 } & Negative & 30 & 1 & & & & & \\
\hline & Positive & 112 & 1.5 & $0.65-3.5$ & 0.34 & & & \\
\hline Basal phenotype marker & Negative & 9 & 1 & & & & & \\
\hline (CK5/6, CK14, EGFR) & Positive & 133 & 1.0 & $0.24-4.3$ & 0.98 & & & \\
\hline \multirow[t]{2}{*}{ P-cadherin } & Low & 90 & 1 & & & & & \\
\hline & High & 52 & 1.3 & $0.59-2.8$ & 0.52 & & & \\
\hline \multirow[t]{2}{*}{ Tumor size } & $<5.0 \mathrm{~cm}$ & 17 & 1 & & & 1 & & \\
\hline & $\geqq 5.0 \mathrm{~cm}$ & 122 & 4.9 & $2.1-11.4$ & 0.0003 & 5.7 & $2.3-14.1$ & 0.0002 \\
\hline \multirow[t]{2}{*}{ Lymph-node metastasis } & $(-)$ & 80 & 1 & & & & & \\
\hline & $(+)$ & 59 & 3.8 & $1.6-8.8$ & 0.0020 & & & \\
\hline \multirow[t]{2}{*}{ Nuclear grade } & 1,2 & 111 & 1 & & & & & \\
\hline & 3 & 31 & 4.2 & $1.9-9.0$ & 0.0002 & & & \\
\hline \multirow[t]{2}{*}{ Chemotherapy } & No & 93 & 1 & & & 1 & & \\
\hline & Yes & 49 & 2.6 & $1.2-5.7$ & 0.014 & 3.5 & $1.5-7.9$ & 0.0028 \\
\hline
\end{tabular}

Abbreviation: 95\%Cl 95\% confidence interval, LI labeling index.

negative tumors, and Ki67-high and p53-positive tumors) in both the DFS $(P<0.0001, P<0.0001$, and $P=0.0005$, respectively; Figure $3 \mathrm{~A})$ and $\mathrm{OS}(P=0.0010, P=0.011$, and $P<0.0001$, respectively; Figure $3 \mathrm{~B}$ ) analyses. The DFS and OS curves therefore differed significantly between the patients with favorable-phenotype tumors and those with unfavorable-phenotype tumors (DFS: HR, 9.3; 95\% CI, 3.5-24.5; log-rank $P<0.0001$; Figure 3C, and OS: HR, 8.8; 95\% CI, 1.9-40.4; $\log$-rank $P=0.0007$; Figure 3D). This difference was much more distinct than that between the low- and high-Ki67-LI curves (DFS: HR, 3.6; 95\% CI, 1.77.9, and OS: HR, 4.4; 95\% CI, 1.3-14.6). The 5- and 10year OS rates were $97 \%$ and $97 \%$, respectively, for the patients with favorable-phenotype tumors but only $91 \%$ and $65 \%$, respectively, for the patients with unfavorablephenotype tumors.

We next conducted subgroup analysis of HR-positive and HER2-negative breast cancer patients who had and had not received pre- or post-operative chemotherapy. Among the 49 patients who had received chemotherapy, those with favorable-phenotype tumors had significantly longer DFS than those with unfavorable-phenotype tumors $(P<0.0001)$. And then, among the 93 patients who had not received chemotherapy, those with favorable-phenotype tumors had significantly or almost significantly longer DFS than those with unfavorablephenotype tumors $(P=0.0002)$.
Finally, we performed multivariate analyses of survival using Cox's model of the proportional hazards regression including immunohistochemical parameters (combined Ki67-p53 status, FOXA1, GATA3, basal phenotype marker and P-cadherin) and the established clinicopathological factors (tumor size, lymph-node metastasis, nuclear grade and chemotherapy).

In those analyses, combined Ki67-p53 status, tumor size and chemotherapy was a significant prognostic indicators of DFS $(P<0.0001, P=0.0001$ and $P=0.0028$, respectively; Table 3) and combined Ki67-p53 status was an only significant prognostic indicator of OS $(P=0.0081$, Table 4$)$.

\section{Discussion}

The international expert panel at the 2009 St. Gallen Consensus meeting referred to the importance of proliferation markers in deciding whether to include adjuvant chemotherapy in the treatment of patients with HRpositive HER-2-negative breast cancers. Several largescale studies have evaluated the clinical significance of the Ki67 LI among patients with HR-positive breast cancer $[7,18,21]$. In this study, we showed that an IHC panel comprising p53 status and Ki67 LI is more accurate than Ki67 LI alone at predicting the prognosis for patients with HR-positive and HER2-negative breast cancer.

The results of our IHC panel divided the patients with HR-positive and HER2-negative invasive breast cancers into 
Table 4 Univariate and multivariate analyses of overall survival in patients with hormone receptor-positive/HER2negative primary breast cancer

\begin{tabular}{|c|c|c|c|c|c|c|c|c|}
\hline & & \multirow[b]{2}{*}{$\begin{array}{c}\text { Total } \\
(n=142)\end{array}$} & \multicolumn{3}{|c|}{ Univariate } & \multicolumn{3}{|c|}{ Multivariate } \\
\hline & & & $\begin{array}{c}\text { Hazard } \\
\text { ratio }\end{array}$ & $(95 \% \mathrm{Cl})$ & $P$-value & $\begin{array}{c}\text { Hazard } \\
\text { ratio }\end{array}$ & $(95 \% \mathrm{Cl})$ & $P$-value \\
\hline \multirow[t]{2}{*}{ Ki67-p53 } & Low Ki67 LI and Negative p53 & 88 & 1 & & & 1 & & \\
\hline & High Ki67 LI and/or Positive p53 & 54 & 8.8 & $1.9-40.4$ & 0.0049 & 7.9 & $1.7-36.7$ & 0.0081 \\
\hline \multirow[t]{2}{*}{ FOXA1 } & Low & 20 & 1 & & & & & \\
\hline & High & 119 & 0.49 & $0.06-3.9$ & 0.50 & & & \\
\hline \multirow[t]{2}{*}{ GATA3 } & Negative & 30 & 1 & & & & & \\
\hline & Positive & 112 & 1.1 & $0.31-4.3$ & 0.84 & & & \\
\hline Basal phenotype marker & Negative & 9 & 1 & & & & & \\
\hline (CK5/6, CK14, EGFR) & Positive & 133 & 1.2 & $0.15-9.3$ & 0.86 & & & \\
\hline \multirow[t]{2}{*}{ P-cadherin } & Low & 90 & 1 & & & & & \\
\hline & High & 52 & 1.8 & $0.57-5.5$ & 0.32 & & & \\
\hline \multirow[t]{2}{*}{ Tumor size } & $<5.0 \mathrm{~cm}$ & 17 & 1 & & & & & \\
\hline & $\geqq 5.0 \mathrm{~cm}$ & 122 & 3.4 & $0.90-12.8$ & 0.071 & & & \\
\hline \multirow[t]{2}{*}{ Lymph-node metastasis } & $(-)$ & 80 & 1 & & & & & \\
\hline & $(+)$ & 59 & 3.9 & $1.02-14.7$ & 0.045 & & & \\
\hline \multirow[t]{2}{*}{ Nuclear grade } & 1,2 & 111 & 1 & & & & & \\
\hline & 3 & 31 & 3.8 & $1.2-11.9$ & 0.0020 & & & \\
\hline \multirow[t]{2}{*}{ Chemotherapy } & No & 93 & 1 & & & & & \\
\hline & Yes & 49 & 2.0 & $0.65-6.3$ & 0.22 & & & \\
\hline
\end{tabular}

Abbreviation: 95\% Cl 95\% confidence interval, Ll labeling index.

2 distinct prognostic subtypes, those with favorablephenotype (Ki67 LI-low and p53-negative) and unfavorablephenotype group (Ki67 LI-high and/or p53-positive) tumors. Multivariate analysis showed that the IHC panel results, tumor size and chemotherapy were independent prognostic factors for DFS and that the IHC panel results was an only independent prognostic factor for OS. Furthermore, 76 of the 78 patients (97\%) with early-clinical-stage (I or II) cancers showing the favorable phenotype were alive at the end of this study. The results of a similar immunohistochemical biomarker panel for 6 markers, including p53 and Ki67, were reported by Brian et al. to be a significant prognostic factor [32]. Ross et al. also showed that the immunohistochemical detection of 5 markers, including p53, was significantly associated with clinical outcome [33]. These reports support our data, at least in part; moreover, our immunohistochemical panel using 2 easy-to-use antibodies (Ki67 and anti-p53 antibodies) was both simpler than the cited panels. Miller et al. reported the similar results to this reports [24]. They evaluated the three molecular marker (Ki67, p53 and HER2) using the whole cases with HRpositive tumors. On the other hand, we excluded the HER2positive tumors from the whole HR-positive tumors and then evaluate the clinicopathological implication of combined Ki67-p53 status in the patients with HR-positive and HER2-negative tumors. Nowadays, the patients with HER2- positive tumors are treated with anti-HER2 drugs and show different clinical outcome to those with HER2-negative tumors. So, our results give the more precise information and are more applicable to the dairy practice than the results of Miller et al.

The results of the immunohistochemical panel divided the HR-positive and HER2-negative breast cancer patients as follows: the 10 -year DFS rates were $81 \%$ for the favorable phenotype group and $46 \%$ for the unfavorable phenotype group, while the 10 -year OS rates were $97 \%$ and $65 \%$ for the favorable and unfavorable phenotype groups, respectively. To exclude the influence of adjuvant chemotherapy on the predictive value of the panel, we examined the prognostic significance of the panel separately in patients who received either pre- or post- operative chemotherapy and those who received no chemotherapy. As the immunohistochemical panel results were also identified as a significant prognostic factor in the patients who did not receive chemotherapy, we were able to exclude the influence of chemotherapy on our results. Our data indicate that patients with favorable-phenotype cancers have a clinical choice to avoid cytotoxic chemotherapy, as the baseline prognosis with adjuvant hormonal therapy alone is very good for this group.

In this report, the unfavorable phenotype-tumors exhibited significantly higher rates of positivity for 
HER2, basal phenotype markers (CK5/6, CK14, and EGFR), and P-cadherin than did the favorable-phenotype tumors. P-cadherin has been previously shown to be overexpressed on basal-type tumors $[14,34]$. These properties suggest that unfavorable-phenotype tumors take on not only the "HER2" phenotype [7] but also the "basal" phenotype. To our knowledge, this is the first clinical study to reveal an obvious correlation between luminal subtype-B and basal-type breast tumors. Basaltype tumors exhibit more $p 53$ mutations $[9,35]$ and nuclear p53 protein accumulation [36] than do luminaltype or HER2-type tumors, so $p 53$ mutation is thought to be one of the characteristics of basal-type tumors $[37,38]$. Our results would be consistent with this viewpoint. We suspect that some tumors should be considered "mixed intrinsic subtype" tumors, that is, tumors that exhibit characteristics of 2 or more intrinsic subtypes and therefore cannot be classified as any "pure" intrinsic subtype.

\section{Conclusions}

In conclusion, our results revealed that the cases with Ki67 LI-high and p53 positive showed a mixed tendency towards the "HER2" and "basal" types ,and that a simple immunohistochemical panel comprising Ki67 and p53 could distinguish between the cases with a favorable phenotype group and those with an unfavorable phenotype group among HR-positive and HER2-negative breast cancer patients. These suggest that our simple immunohistochemical panel comprising Ki67 and p53 is a promising tool for distinguishing between "luminalsubtype-A" and "luminal-subtype-B" breast cancers and management of patients with HR-positive breast cancer.

\section{Abbreviations \\ Ki67 LI: Ki67 labeling index; IHC panel: Immunohistochemical panel; HR: Hormone receptor; ER: Estrogen receptor; PgR: Progesterone receptor; TMA: Tissue microarray.}

\section{Competing interests}

The authors have declared no conflicts of interest.

\section{Authors' contributions}

TK and $\mathrm{KI}$ conceived of the study, performed experiments, analyzed data and wrote the manuscript. TM, TY and JY provided samples, collected clinical and pathological data. HT participated in designing the study and revising the manuscript. OM participated in the overall design, study coordination and finalized the draft of the manuscript. All authors read and approved the final manuscript.

\section{Acknowledgment}

This work was supported by the Foundation for Promotion of Defense Medicine.

\section{Author details}

${ }^{1}$ Department of Basic Pathology, National Defense Medical College, 3-2 Namiki, Tokorozawa, Saitama 359-8513, Japan. ²Department of Surgery, National Defense Medical College, 3-2 Namiki, Tokorozawa, Saitama 359-8513, Japan. ${ }^{3}$ Pathology and Clinical Laboratory Division, National Cancer Center Hospital, 5-1-1 Tsukiji, Chuo-ku, Tokyo 104-0045, Japan.
Received: 2 October 2012 Accepted: 30 January 2013

Published: 6 February 2013

\section{References}

1. Badve S, Nakshatri H: Oestrogen-receptor-positive breast cancer: towards bridging histopathological and molecular classifications. J Clin Pathol 2009, 62(1):6-12

2. Hortobagyi GN: Treatment of breast cancer. N Engl J Med 1998, 339(14):974-984.

3. Early Breast Cancer Trialists' Collaborative Group: Tamoxifen for early breast cancer: an overview of the randomised trials. Lancet 1998 351(9114):1451-1467.

4. Mauri D, Pavlidis N, Polyzos NP, loannidis JP: Survival with aromatase inhibitors and inactivators versus standard hormonal therapy in advanced breast cancer: meta-analysis. J Nat/ Cancer Inst 2006, 98 (18):1285-1291.

5. Fisher B, Jeong JH, Bryant J, Anderson S, Dignam J, Fisher ER, Wolmark N: Treatment of lymph-node-negative, oestrogen-receptor-positive breast cancer: long-term findings from National Surgical Adjuvant Breast and Bowel Project randomised clinical trials. Lancet 2004, 364(9437):858-868.

6. Paik S, Shak S, Tang G, Kim C, Baker J, Cronin M, Baehner FL, Walker MG, Watson $D$, Park T, et al: A multigene assay to predict recurrence of tamoxifen-treated, node-negative breast cancer. N Engl I Med 2004, 351(27):2817-2826

7. Cheang MC, Chia SK, Voduc D, Gao D, Leung S, Snider J, Watson M, Davies S, Bernard PS, Parker JS, et al: Ki67 index, HER2 status, and prognosis of patients with luminal B breast cancer. J Natl Cancer Inst 2009, 101(10):736-750

8. Paik S, Tang G, Shak S, Kim C, Baker J, Kim W, Cronin M, Baehner FL, Watson $D$, Bryant J, et al: Gene expression and benefit of chemotherapy in women with node-negative, estrogen receptor-positive breast cancer. J Clin Oncol 2006, 24(23):3726-3734.

9. Sorlie T, Perou CM, Tibshirani R, Aas T, Geisler S, Johnsen H, Hastie T, Eisen $M B$, van de Rijn $M$, Jeffrey $S S$, et al: Gene expression patterns of breast carcinomas distinguish tumor subclasses with clinical implications. Proc Natl Acad Sci USA 2001, 98(19):10869-10874.

10. Lacroix M, Toillon RA, Leclercq G: p53 and breast cancer, an update. Endocr Relat Cancer 2006, 13(2):293-325.

11. Calza S, Hall P, Auer G, Biohle J, Klaar S, Kronenwett U, Liu ET, Miller L, Ploner A, Smeds J, et al: Intrinsic molecular signature of breast cancer in a population-based cohort of 412 patients. Breast Cancer Res 2006, 8(4):R34.

12. Novelli F, Milella M, Melucci E, Di Benedetto A, Sperduti I, Perrone-Donnorso R, Perracchio L, Venturo I, Nistico C, Fabi A, et al: A divergent role for estrogen receptor-beta in node-positive and node-negative breast cancer classified according to molecular subtypes: an observational prospective study. Breast Cancer Res 2008, 10(5):R74.

13. Hu Z, Fan C, Oh DS, Marron JS, He X, Qaqish BF, Livasy C, Carey LA, Reynolds $E$, Dressler $L$, et al: The molecular portraits of breast tumors are conserved across microarray platforms. BMC Genomics 2006, 7:96.

14. Wirapati P, Sotiriou C, Kunkel S, Farmer P, Pradervand S, Haibe-Kains B, Desmedt C, Ignatiadis M, Sengstag T, Schutz F, et al: Meta-analysis of gene expression profiles in breast cancer: toward a unified understanding of breast cancer subtyping and prognosis signatures. Breast Cancer Res 2008, 10(4):R65.

15. Iwaya K, Tsuda H, Hiraide H, Tamaki K, Tamakuma S, Fukutomi T, Mukai K Hirohashi S: Nuclear p53 immunoreaction associated with poor prognosis of breast cancer. Jpn J Cancer Res 1991, 82(7):835-840.

16. Olivier M, Langerod A, Carrieri P, Bergh J, Klaar S, Eyfjord J, Theillet C, Rodriguez C, Lidereau R, Bieche I, et al: The clinical value of somatic TP53 gene mutations in 1,794 patients with breast cancer. Clin Cancer Res 2006, 12(4):1157-1167.

17. Thorat MA, Marchio C, Morimiya A, Savage K, Nakshatri H, Reis-Filho JS, Badve S: Forkhead box A1 expression in breast cancer is associated with luminal subtype and good prognosis. J Clin Pathol 2008, 61(3):327-332.

18. Jacquemier J, Charafe-Jauffret E, Monville F, Esterni B, Extra JM, Houvenaeghel G, Xerri L, Bertucci F, Birnbaum D: Association of GATA3, P53, Ki67 status and vascular peritumoral invasion are strongly prognostic in luminal breast cancer. Breast Cancer Res 2009, 11 (2):R23.

19. Yerushalmi R, Woods R, Ravdin PM, Hayes MM, Gelmon KA: Ki67 in breast cancer: prognostic and predictive potential. Lancet Oncol 2010, 11(2):174-183. 
20. Brown DC, Gatter KC: Ki67 protein: the immaculate deception? Histopathology 2002, 40(1):2-11.

21. Viale G, Giobbie-Hurder A, Regan MM, Coates AS, Mastropasqua MG, Dell'Orto P, Maiorano E, MacGrogan G, Braye SG, Ohlschlegel C, et al: Prognostic and predictive value of centrally reviewed Ki-67 labeling index in postmenopausal women with endocrine-responsive breast cancer: results from Breast International Group Trial 1-98 comparing adjuvant tamoxifen with letrozole. J Clin Oncol 2008, 26(34):5569-5575.

22. Kobayashi T, Tsuda H, Moriya T, Yamasaki T, Kikuchi R, Ueda S, Omata J, Yamamoto J, Matsubara O: Expression pattern of stromal cell-derived factor-1 chemokine in invasive breast cancer is correlated with estrogen receptor status and patient prognosis. Breast Cancer Res Treat 2010, 123(3):733-745.

23. Sakamoto G, Inaji H, Akiyama F, Haga S, Hiraoka M, Inai K, Iwase T, Kobayashi S, Sano M, Sato T, et al: General rules for clinical and pathological recording of breast cancer 2005. Breast Cancer 2005, 12(Suppl):S1-S27.

24. Millar EK, Graham PH, McNeil CM, Browne L, O'Toole SA, Boulghourjian A, Kearsley JH, Papadatos G, Delaney G, Fox C, et al: Prediction of outcome of early ER+ breast cancer is improved using a biomarker panel, which includes Ki-67 and p53. Br J Cancer 2011, 105(2):272-280.

25. Bago-Horvath Z, Rudas M, Dubsky P, Jakesz R, Singer CF, Kemmerling R, Greil R, Jelen A, Bohm G, Jasarevic Z, et al: Adjuvant sequencing of tamoxifen and anastrozole is superior to tamoxifen alone in postmenopausal women with low proliferating breast cancer. Clin Cancer Res 2011, 17(24):7828-7834

26. Alba E, Calvo L, Albanell J, De la Haba JR, Arcusa Lanza A, Chacon JI, SanchezRovira P, Plazaola A, Lopez Garcia-Asenjo JA, Bermejo B, et al: Chemotherapy (CT) and hormonotherapy $(\mathrm{HT})$ as neoadjuvant treatment in luminal breast cancer patients: results from the GEICAM/2006-03, a multicenter, randomized, phase-II study. Ann Oncol 2012, 23(12):3069-3074.

27. Domagala W, Markiewski M, Harezga B, Dukowicz A, Osborn M: Prognostic significance of tumor cell proliferation rate as determined by the MIB-1 antibody in breast carcinoma: its relationship with vimentin and p53 protein. Clin Cancer Res 1996, 2(1):147-154.

28. Pinto AE, Andre S, Laranjeira C, Soares J: Correlations of cell cycle regulators (p53, p21, pRb and $\mathrm{mdm} 2$ ) and c-erbB-2 with biological markers of proliferation and overall survival in breast cancer. Pathology 2005, 37(1):45-50.

29. Wolff AC, Hammond ME, Schwartz JN, Hagerty KL, Allred DC, Cote RJ, Dowsett M, Fitzgibbons PL, Hanna WM, Langer A, et al: American Society of Clinical Oncology/College of American Pathologists guideline recommendations for human epidermal growth factor receptor 2 testing in breast cancer. J Clin Oncol 2007, 25(1):118-145.

30. Perez EA, Romond EH, Suman VJ, Jeong JH, Davidson NE, Geyer CE Jr, Martino S, Mamounas EP, Kaufman PA, Wolmark N: Four-year follow-up of trastuzumab plus adjuvant chemotherapy for operable human epidermal growth factor receptor 2-positive breast cancer: joint analysis of data from NCCTG N9831 and NSABP B-31. J Clin Oncol 2011, 29(25):3366-3373.

31. Slamon DJ, Leyland-Jones B, Shak S, Fuchs H, Paton V, Bajamonde A, Fleming T, Eiermann W, Wolter J, Pegram M, et al: Use of chemotherapy plus a monoclonal antibody against HER2 for metastatic breast cancer that overexpresses HER2. N Engl J Med 2001, 344(11):783-792.

32. Ring BZ, Seitz RS, Beck R, Shasteen WJ, Tarr SM, Cheang MC, Yoder BJ, Budd GT, Nielsen TO, Hicks DG, et al: Novel prognostic immunohistochemical biomarker panel for estrogen receptor-positive breast cancer. J Clin Oncol 2006, 24(19):3039-3047.

33. Ross DT, Kim CY, Tang G, Bohn OL, Beck RA, Ring BZ, Seitz RS, Paik S, Costantino JP, Wolmark N: Chemosensitivity and stratification by a five monoclonal antibody immunohistochemistry test in the NSABP B14 and B20 trials. Clin Cancer Res 2008, 14(20):6602-6609.

34. Matos I, Dufloth R, Alvarenga M, Zeferino LC, Schmitt F: p63, cytokeratin 5 , and P-cadherin: three molecular markers to distinguish basal phenotype in breast carcinomas. Virchows Arch 2005, 447(4):688-694.

35. Carey LA, Perou CM, Livasy CA, Dressler LG, Cowan D, Conway K, Karaca G, Troester MA, Tse CK, Edmiston S, et al: Race, breast cancer subtypes, and survival in the Carolina Breast Cancer Study. JAMA 2006, 295(21):2492-2502.

36. Tan DS, Marchio C, Jones RL, Savage K, Smith IE, Dowsett M, Reis-Filho JS: Triple negative breast cancer: molecular profiling and prognostic impact in adjuvant anthracycline-treated patients. Breast Cancer Res Treat 2008, 111(1):27-44.
37. Brenton JD, Carey LA, Ahmed AA, Caldas C: Molecular classification and molecular forecasting of breast cancer: ready for clinical application? J Clin Oncol 2005, 23(29):7350-7360.

38. Schneider BP, Winer EP, Foulkes WD, Garber J, Perou CM, Richardson A, Sledge GW, Carey LA: Triple-negative breast cancer: risk factors to potential targets. Clin Cancer Res 2008, 14(24):8010-8018.

doi:10.1186/1472-6890-13-5

Cite this article as: Kobayashi et al:. A simple immunohistochemical panel comprising 2 conventional markers, Ki67 and p53, is a powerful tool for predicting patient outcome in luminal-type breast cancer. BMC Clinical Pathology 2013 13:5.

\section{Submit your next manuscript to BioMed Central and take full advantage of:}

- Convenient online submission

- Thorough peer review

- No space constraints or color figure charges

- Immediate publication on acceptance

- Inclusion in PubMed, CAS, Scopus and Google Scholar

- Research which is freely available for redistribution 